\section{RIMAPS and Variogram Analysis of Barley Leaf Surfaces}

Eduardo A. Favret ${ }^{*} \dagger$ and Néstor O. Fuentes ${ }^{*} \dagger$

* Comisión Nacional de Energía Atómica, Buenos Aires, Argentina

* Instituto de Tecnología "Prof. Jorge A. Sábato", Univer-

sidad Nacional de Gral. San Martin. Buenos Aires, Argentina favret@cnea.gov.ar

\section{Introduction}

It is a common practice to use microscopic images to describe the differences observed between plant tissues. The images illustrate the taxonomic characteristics of the studied species. In this work we introduce a quantitative method for conducting these analyses

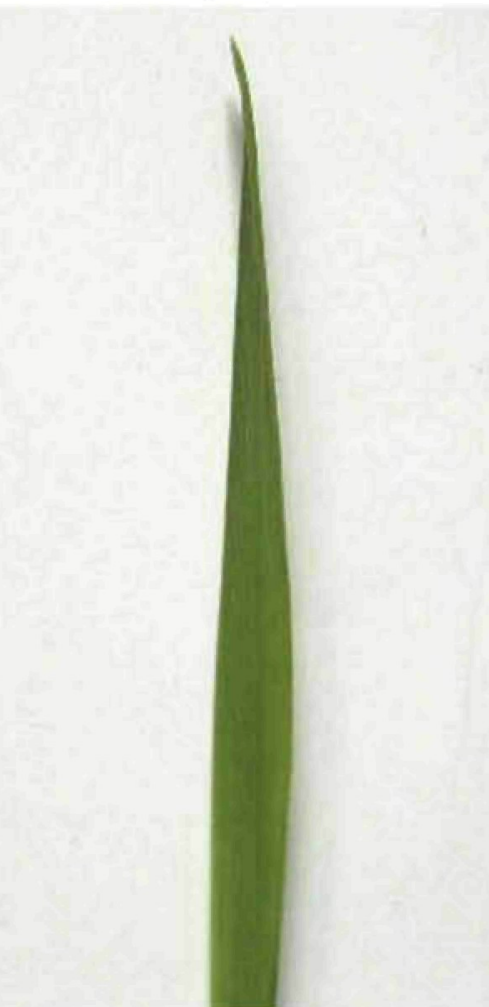
leaf.

Figure 1: Macrograph of a barley confirms the existence of the Golden Ratio value of 1.618. The same value is verified when determining the ratio between the width of obtained via scanning elecbarley leaf surfaces. The topography of the leaf surfaces of a narrow-leaf mutant and its wild type mother line was characterized, see figure 1 , using the Rotated Image with Maximum Average Power Spectrum (RIMAPS) technique and the Variogram method. Spectra resulting from RIMAPS analysis allow us to identify the specimens and to distinguish between the adaxial or the abaxial side of the leaf. These results are complemented by obtaining the typical scale lengths that characterize the abaxial surfaces of both the mutant and the mother line barley leaves. Comparisons between the widths of long cells on the two specimens the mother line leaf and the mutant barley leaf.

utilizing digitized images tron microscopy (SEM) of

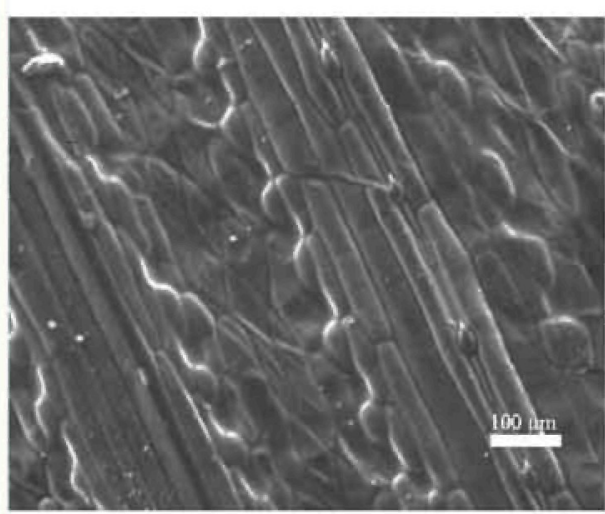

Figure 2: SEM micrograph of a barley leaf (Mother line). Adaxial surface.

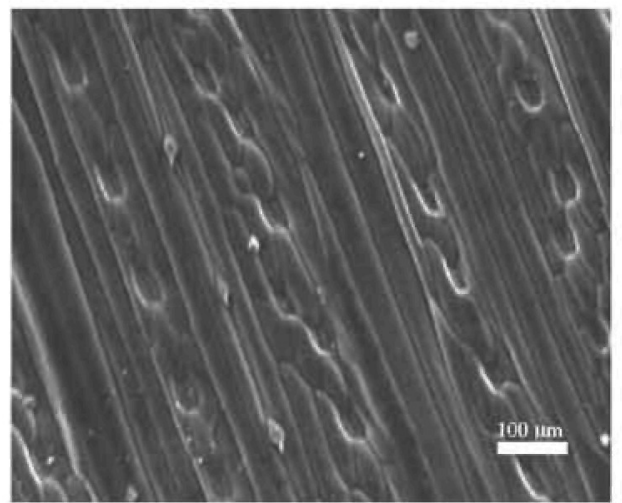

Figure 3: SEM micrograph of a barley leaf (Mother line). Abaxial surface.
It is well known that the Golden Ratio is found in nature [1-2]. One example is in connection with the Fibonacci sequence. This sequence describes a pattern that may be observed in many situations involving growth, from the growth of plants to the growth of a computer database. The ratio of successive terms of the Fibonacci sequence, as the number of terms becomes larger, gets closer and closer to the Golden Ratio value of 1.618.

\section{RIMAPS}

RIMAPS is a surface characterization technique that uses digitized images and is independent of the class of microscopy (Light Microscopy, Electron Microscopy) and of conditions used for observation (Bright Field Illumination, Dark Field Illumination, magnification, contrast, etc.). This technique consists basically in performing the following steps:

- rotation of the original image from $0^{\circ}$ to $360^{\circ}$ by step increments $\alpha$;

- calculation of the integral of variable $x$ of the two-dimensional Fourier transform for each $y$-line of the new image obtained after rotation;

- computation of the average power spectra for each angular position;

- plotting of the maximum values of the power spectra as a function of the angle of rotation.

The peaks appearing in the resulting plots indicate the orientation of surface micropatterns and their characteristic topographic form [3-6]. By means of this technique, the orientation and characteristics of the leaf surface topography can be determined. In many cases, surface patterns can be associated with simple geometrical figures such as lines, squares or triangles, distributed with a certain orientation on the surface [3-6].

\section{Variogram}

A variogram is the name of a mathematical method for the quantification of length scale-dependent topography. From a given digitized image of a surface, the root mean square (rms) parameter is calculated for all the possible areal windows with different area sizes on a surface. The calculated rms parameters are then represented on a log-log plot as a function of the window area. Intersections of different slopes in the plot give the crossover lengths that represent the characteristic scale lengths of the surface [6].

\section{Experimental procedure}

Observations of the first leaf surface of 14-day old barley seedlings were carried out with an SEM. To investigate epidermal relief, the following preparation techniques were applied: liquid substitution with glycerol [7], without coating, of fresh samples that had

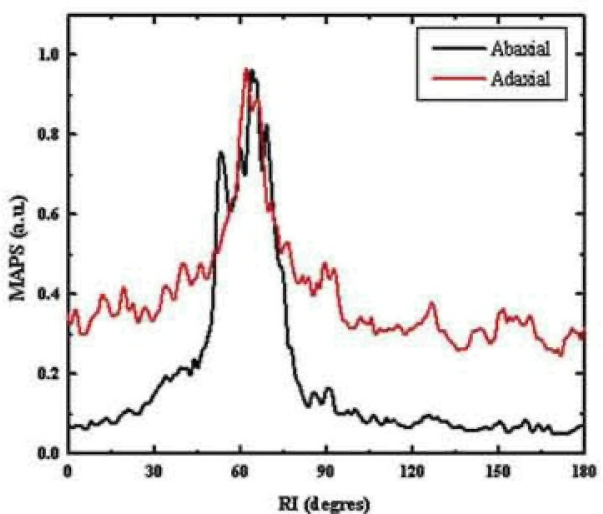

Figure 4: RIMAPS spectra of the digitized images observed in figures 2 and 3 . 


\section{See More. Guess Less.}
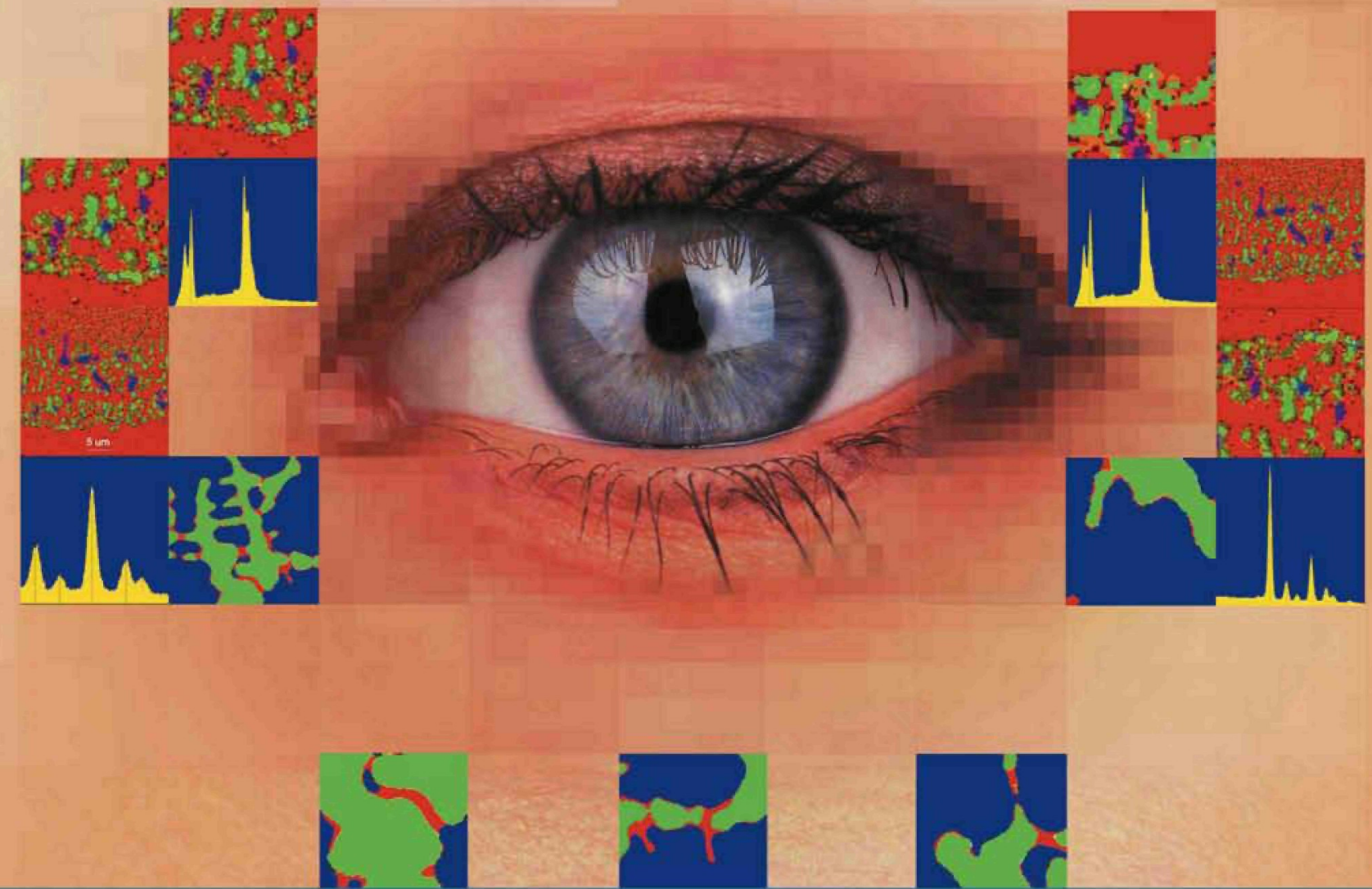

\section{NORAN System SIX for x-ray microanalysis}

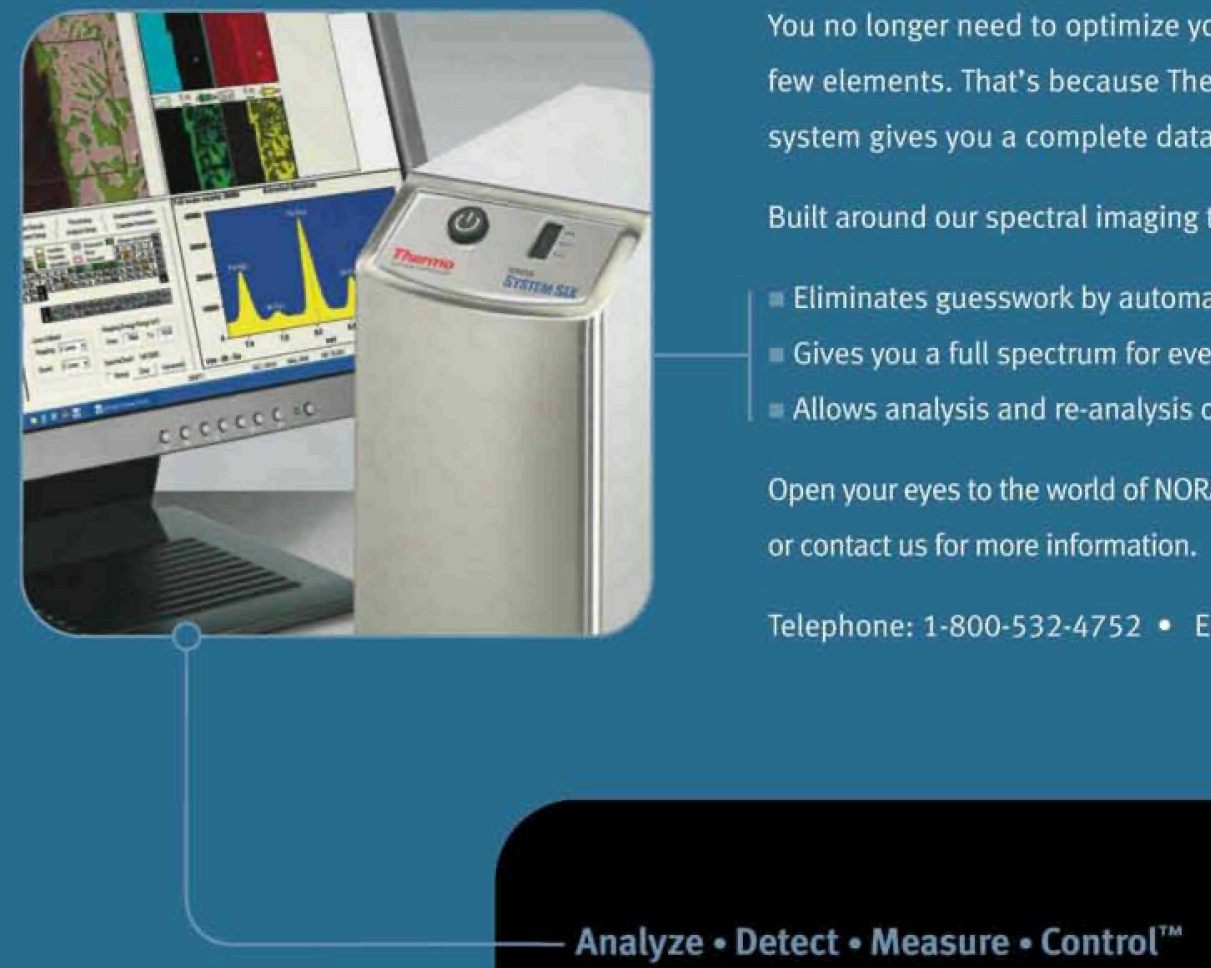




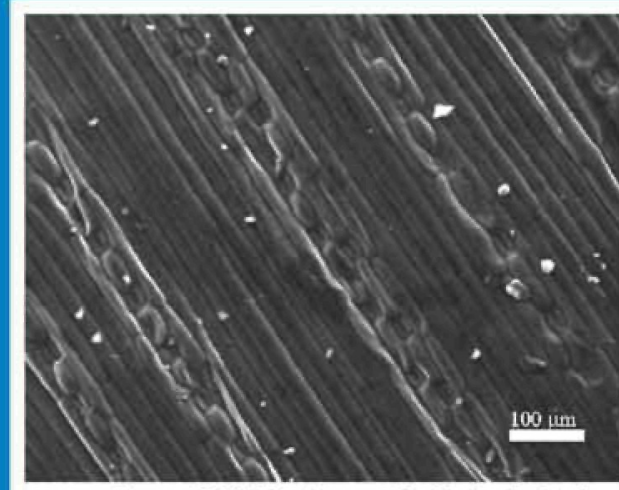

Figure 5: SEM micrograph of a barley leaf

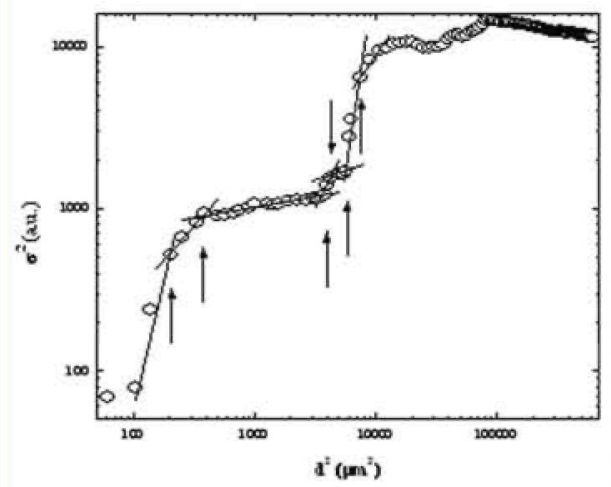

Figure 8: Variogram of the digitized image observed in figure 5. Arrows indicate crossover lengths. (Mutant). Abaxial surface.

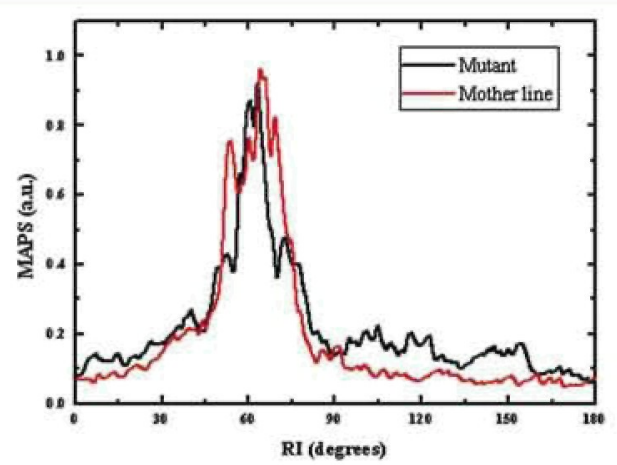

Figure 6: RIMAPS spectra of the digitized images observed in figures 3 and 5 .

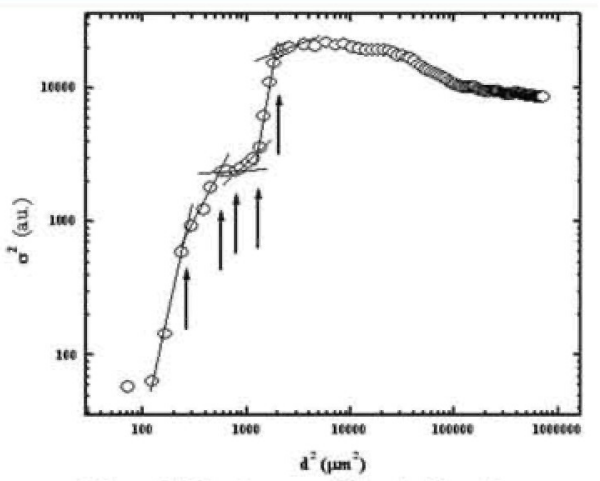

Figure 7: Variogram of the digitized image observed in figure 3. Arrows indicate crossover lengths.

Variogram analysis of the abaxial surfaces (first leaf) of mother been previously fixed with formalin-acetic acid-ethanol (FAA).

The main objectives of the liquid substitution method are to stabilize the specimen, to prevent shrinkage, to minimize other artifacts during dehydration, and to render the sample electrically conductive. The specimens are not dried, but their water is instead substituted for a liquid (glycerol) that evaporates very slowly under high vacuum.

\section{Results and discussion}

In the wild type barley, the electron micrographs of both sides of the same leaf show differences between the adaxial (figure 2) and the abaxial (figure 3 ) surfaces. RIMAPS spectra (figure 4) indicate the microstructural differences between both surfaces, given by the distribution, orientation and shape of the cells. A peak at $63^{\circ}$, which corresponds to the apical direction, was common to both surfaces. The adaxial RIMAPS spectrum has a higher background value (base value) than the abaxial RIMAPS spectrum, and also more secondary maxima, produced by a higher area of irregular cells.

When comparison is made between the abaxial surfaces of the wild type and of the narrow-leaf mutant (figure 5) new feature characteristics appear. At first sight, there are a few morphological differences between them:

a) Stings appear in the mother line leaf and not in the mutant,

b) The width of the long cells seems to be lower in the mutant.

The RIMAPS spectra of both surfaces are almost coincident (figure 6), in agreement with previous results, which indicate that RIMAPS spectra identify those peaks representing the biological species [5]. In this case we have the same specie.

When examining the spectra in detail, two main differences are observed:

a) The principal direction $\left(63^{\circ}\right)$ in the mutant is more resolved than in the mother line, which means that the area with long and parallel wall cells is higher,

b) The secondary maxima (far from the main direction) in the mutant have a higher value than in the mother line. lines and mutants (figures 7 and 8 ) gives different crossover lengths. It is an important issue to verify those lengths by looking at the respective micrographs to find out which part of the microstructure represents which topological feature. The results are described in Table I. It is interesting to emphasize the following relation: the quotient between the mean value of the long cell width of the mother line $(32 \mu \mathrm{m})$ and the mutant $(20 \mu \mathrm{m})$ yields 1.6 , approximately the Golden Ratio, and almost the same value is verified when determining the ratio between the width of the mother line first leaf blade (7.8 \pm $0.1 \mathrm{~mm})$ and the narrow leaf mutant $(4.8 \pm 0.1 \mathrm{~mm})$.

The results obtained shows the robustness of RIMAPS and Variogram analyses to distinguish and characterize leaf surfaces. We can identify and quantify the micro-morphological differences between the leaf of a barley mutant and its mother line. Both methods are auxiliary tools for the biologist when he needs to study variations of biological surfaces. The use of RIMAPS and Variograms opens a wide spectrum of possibilities to provide a systematic quantitative

\section{Acknowledgements}

The authors wish to thank Alberto Raul Prina, Instituto de Genética "Ewald A. Favret" CICVyA-INTA Castelar, for providing the barley experimental material and for his valuable suggestions on the manuscript.

Table I Typical Scale Lengths of Barley Leaves

\begin{tabular}{|l|c|c|}
\hline Epidermal structure & Mother Line $[\mu \mathrm{m}]$ & Mutant $[\mu \mathrm{m}]$ \\
\hline Sting width or length & 16 & \\
\hline Stoma width & 24 & 15 \\
\hline Long cell width & 28,36 & 20 \\
\hline Stoma length & 44 & \\
\hline Band width of short cells & 54 & $60-67$ \\
\hline Cell length & 96 & 76,142 \\
\hline Band width of long cells & 114,154 & $88,118,185$ \\
\hline
\end{tabular}

\section{References}

[1] K. Devlin. Mathematics, the Science of Patterns. Scientific American Library. (1994). 108-109.

[2] T. A. Cook. The Curves of Life. Dover Publications. USA. (1979). 81-93 and 414-421.

[3] N. Fuentes and E. Favret. Journal of Microscopy. 206, (2002) 72-83.

[4] E. Favret and N. Fuentes. Materials Characterization. 49 (5) (2003) 387-393.

[5] E. Favret, N. Fuentes and A. Molina, Microscopy and Microanalysis. 2 (Supp]. 2) (2003) 1338-1339.

[6] E. Favret, N. Fuentes and Y. Yu. Applied Surface Science. 230 (2004) 60-72.

[7] H. ]. Hensikat and W. Barthlott. Journal of Microscopy. 172 (1993) 195-203. description of specimens from the study of their leaf surfaces. 
Don't want to miss elements that may be hiding in your sample?

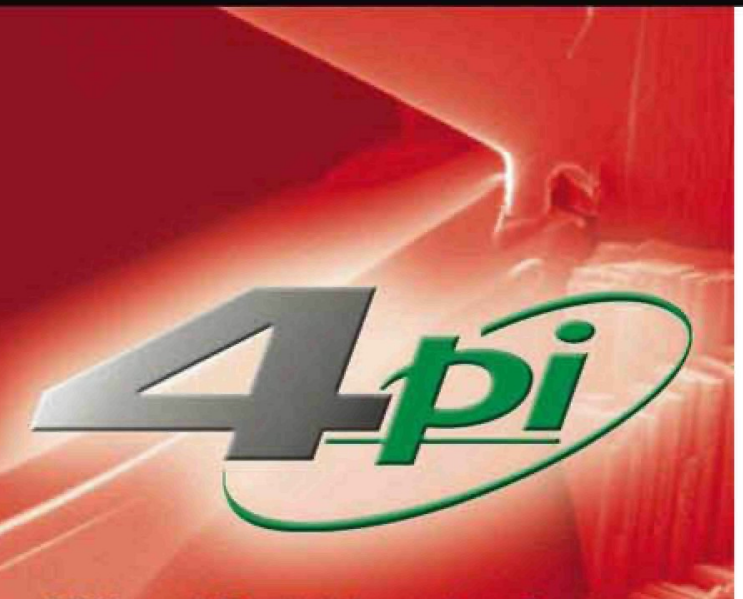

EDX and Digital Imaging Systems

\section{Maximum Pixel Spectrum}

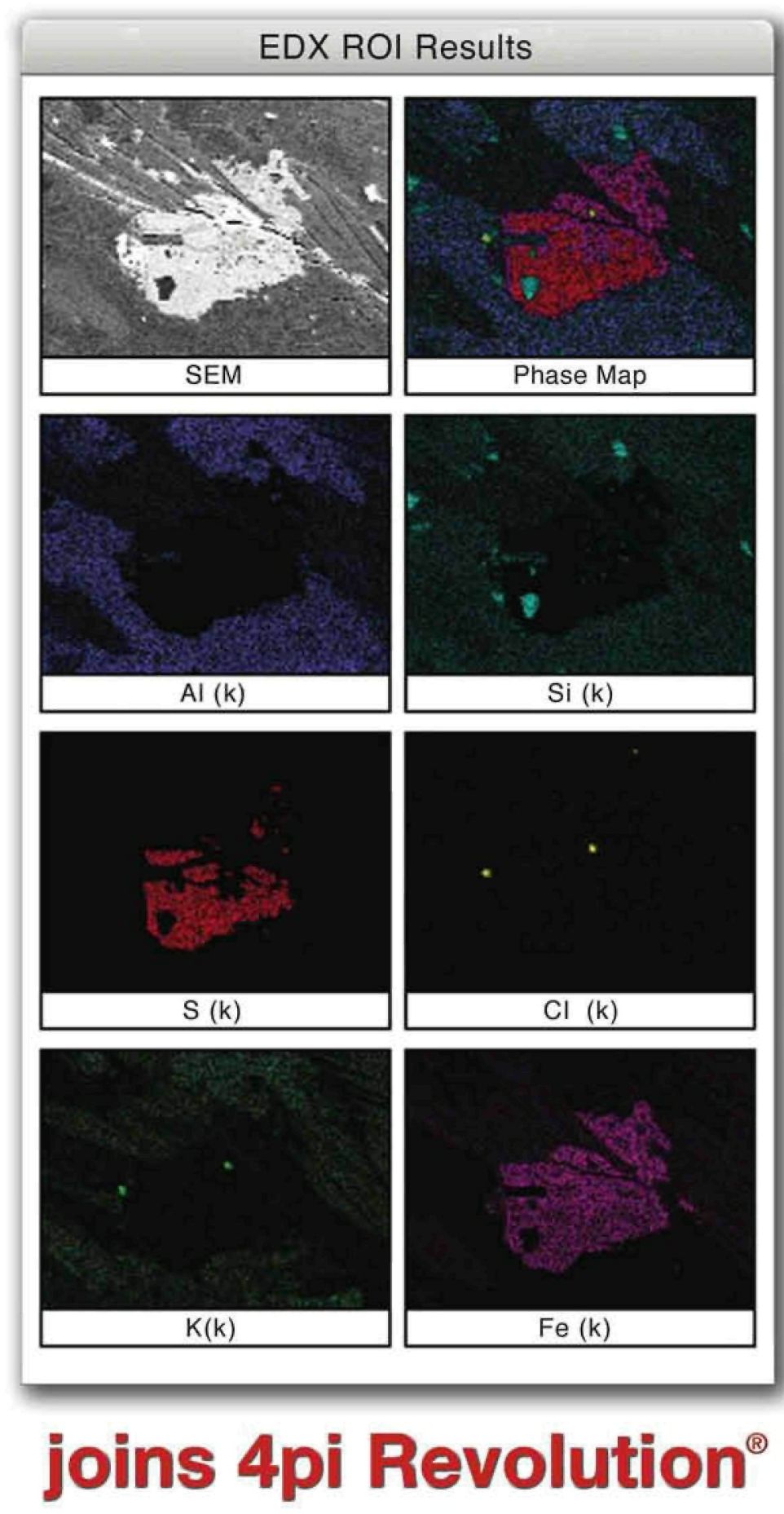

Contact 4pi to ask how its Maximum Pixel Spectrum and Dynamic Element Mapping can benefit your microanalysis results.

4pi Analysis, Inc. • 919-489-1757• info@4pi.com • www.4pi.com 